\title{
Post-Pneumonectomy Changes
}

\author{
Charles-Lwanga Bennin, MD
}

\section{Case}

A 71-year-old woman, with a history of non-small cell lung cancer (NSCLC) with subsequent pneumonectomy presented with worsening dyspnea on exertion and non-productive cough.

The patient first noted the shortness of breath and cough 4 weeks prior to presentation, which were refractory to albuterol nebulization. The patient also reported a $6 \mathrm{lb}$. weight loss during this time period. She denied fevers, chills, night sweats or chest pain. The patient was diagnosed with NSCLC 11 years ago and underwent a right-sided extrapleural pneumonectomy at that time. She received no radiation or chemotherapy. She did not require home oxygen and reported a generally good functional status prior to the development of her latest symptoms. Other past medical history included hyperlipidemia. The patient had been smoking a pack of cigarettes per day for the past 35 years, but denied alcohol or illicit drug use. Current medications included albuterol nebulizer, aspirin, alprazolam, levothyroxine, simvastatin, and over-the-counter Excedrin (acetaminophen, aspirin, caffeine).

On examination, the patient appeared in minimal respiratory distress. She was afebrile, blood pressure was 110/60 mm Hg, the pulse 97 beats per minute, the respiratory rate 16 breaths per minute, and the oxygen saturation $95 \%$ on ambient air. Her heart exam had no murmurs, rubs, or gallops. There were coarse breath sounds over the lower field of the left lung and decreased breath sounds with egophony at the right base. There was no lower extremity edema or calf tenderness. White blood cell count was within normal limits without a bandemia. Chest radiography revealed opacification of the right hemithorax with overall volume loss and left-to-right mediastinal and tracheal

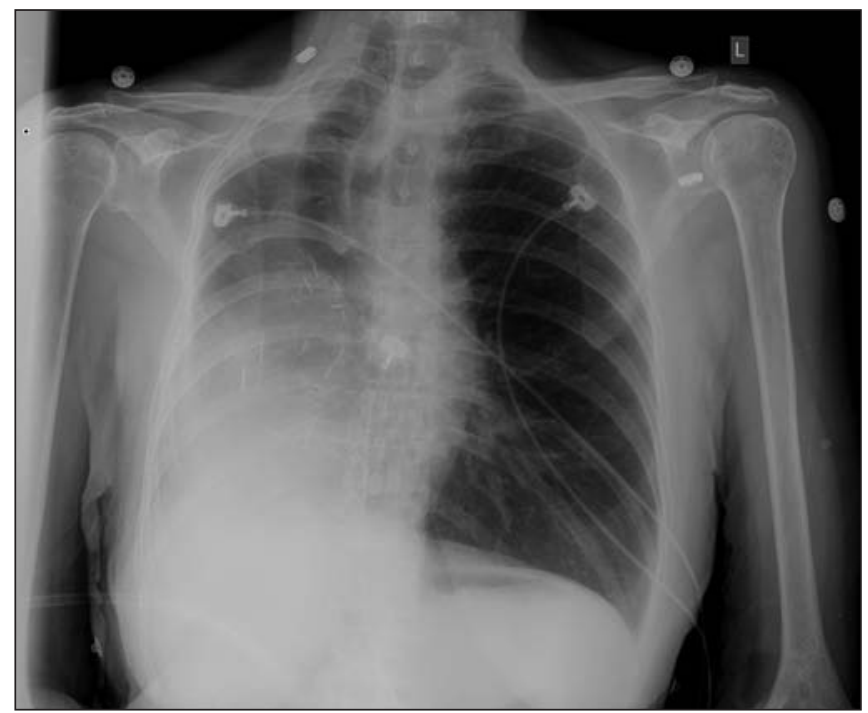

shift in keeping with prior right pneumonectomy. There were several surgical clips seen in the right hemithorax. The left lung field was unremarkable without pneumothorax, pleural effusion, pulmonary edema or focal consolidation. The cardiomediastinal silhouette evaluation was severely limited due to significant left-to-right mediastinal shift. The chest radiograph was interpreted by radiology as "stable post-pneumonectomy changes with no effusions, edema or consolidation." A computed tomography scan was revealed hilar adenopathy with mass effect as well as a new $3 \mathrm{~mm}$ nodule in the left lower lobe.

A diagnosis of small cell cancer was made from bronchoscopy and subsequent tissue biopsy. A nuclear bone scan revealed no metastatic disease and the patient was treated with 3 doses of cisplatin and etoposide. She was discharged home in stable condition.

\section{Discussion}

Pneumonectomy is the surgical removal of a lung. It is indicated in several circumstances including for the removal of tumorous tissue, in occasional settings of traumatic injury, and historically in pulmonary tuberculosis. The removal of a lobe of the lung is termed lobectomy and the removal of a section of the lung is termed wedge resection. The first pneumonectomy dates back to the 1890s when William Macewen performed the procedure in multiple surgical stages. ${ }^{1}$ In 1933, the first single stage en block resection was accomplished by Graham and Singer. ${ }^{2-5}$ Today, there are two surgical types of pneumonectomy performed. A simple pneumonectomy involves the removal of the affected lung only. An extrapleural pneumonectomy involves removal of the affected lung as well as partial resection of the ipsilateral diaphragm, parietal pleura and pericardium.

Predictable anatomical changes occur following pneumonectomy. These changes are especially important when physical findings deviate from the norm. Typically following pneumonectomy, the empty space is filled with air. This space gradually accumulates with fluid over weeks to months. The gradual resorption of air and replacement with fluid eliminates the need for a chest tube post-pneumonectomy. ${ }^{2}$ Complete opacification on chest radiograph eventually occurs in most patients, however a small fraction of patients with post-pneumonectomy will have residual air. ${ }^{5}$

In addition to the gradual accumulation of fluid, the post-pneumonectomy space shrinks, resulting in the elevation of the ipsilateral hemi-diaphragm, shifting of the mediastinum towards the post-pneumonectomy space, and hyperinflation and encroachment of the remaining lung into the post-pneumonectomy space (Figure $2 \& 3$ ).

Figure 1. Upright Xray Chest on admission. 

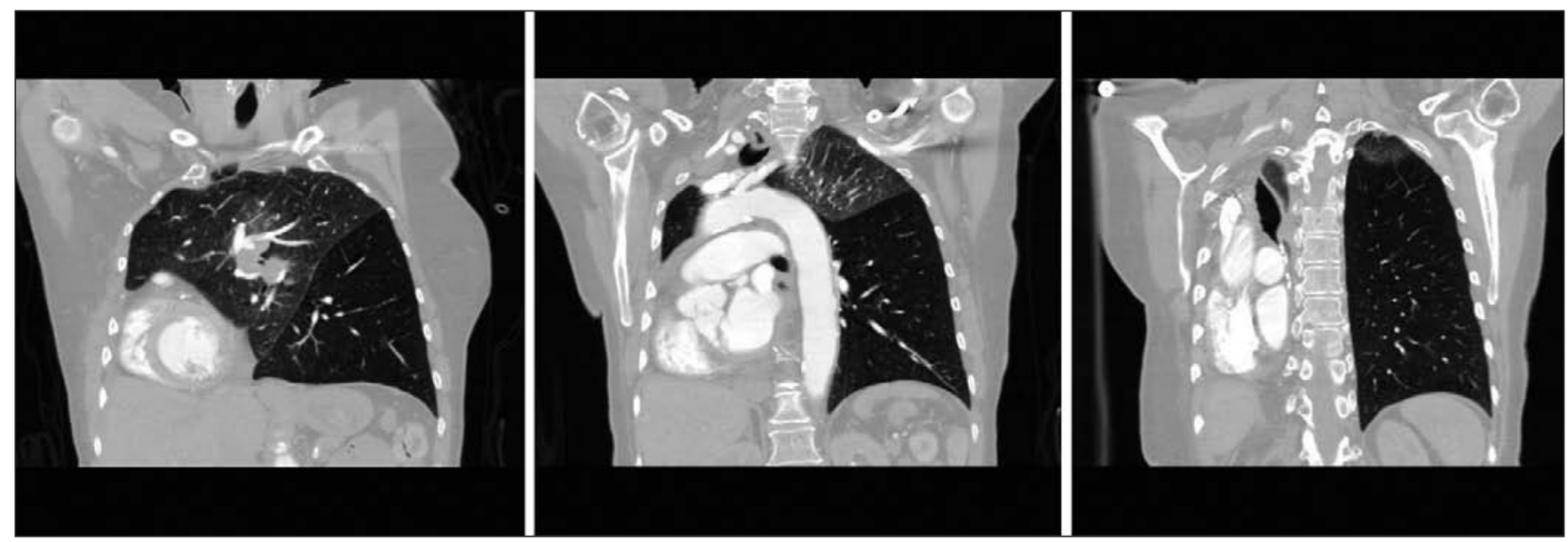

Figure 2. CT Chest; Note the heart shifts into the vacant right pleural space.
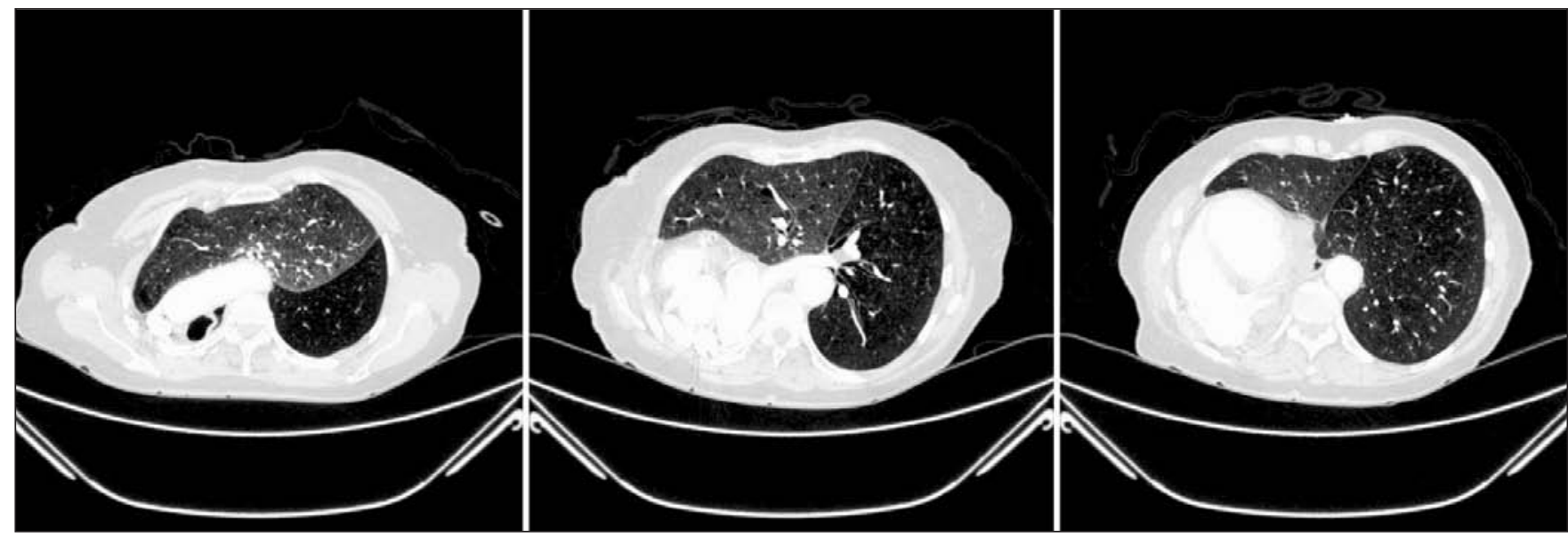

Figure 3. CT Chest; Note the left lung encroaching into the right pleural space.

Although uncommon, fluid can accumulate more rapidly during the first 3 days after surgery. When this occurs, hemothorax and chylothorax must be worked up and ruled out. Rapid post-pneumonectomy edema carries a high mortality rate of more than $50 \%{ }^{6}$

Pneumonectomy has predictable effects on the positions of other vital organs depending on which lung is removed. In a right pneumonectomy, elevation of the right hemi-diaphragm can result in an elevation of the liver into the right post-pneumonectomy space. The heart and great vessels eventually shift into the vacant right post-pneumonectomy space. ${ }^{7}$ These findings are evident on our patient's imaging. In a left pneumonectomy, the heart rotates counterclockwise into the vacant left post-pneumonectomy space. ${ }^{8}$
In light of these anatomical changes, several complications can occur. One complication is a direct result of the severe shifting of the mediastinum, called "post-pneumonectomy syndrome." The surgical incidence for post-pneumonectomy syndrome is 1 in 640 cases, with young age and female sex as strong risk factors. ${ }^{9-12}$ This phenomenon causes large airway obstruction due to the narrowing and stretching of the main bronchus. Symptoms typically present as progressive dyspnea, cough, inspiratory stridor, and recurrent pneumonia ${ }^{5}$. Another complication is the formation of bronchopleural fistulas. The incidence of bronchopleural fistulas associated with pneumonectomy is approximately $5 \%$ with a mortality of 16 $23 \% .^{13}$ The frequency of bronchopleural fistulas is higher for right-sided pneumonectomies. ${ }^{5}$ Symptoms can present as fever, productive cough, hemoptysis, subcutaneous emphysema, 
and persistent air leak from a chest tube. Other complications of pneumonectomy include esophageal fistula, pulmonary edema, arrhythmias and intracardiac shunting. ${ }^{13}$ Chronic pneumonectomy complications include tumor recurrence and emphysema. ${ }^{14}$

\section{Conclusion}

In our case, we present a patient with post-pneumonectomy changes with radiologic findings on imaging. Given her history of right pneumonectomy, these findings were consistent with post-pneumonectomy changes. Incidentally, the patient was also found to have concomitant small cell lung cancer. With an understanding of post-pneumonectomy changes, our patient was correctly diagnosed and appropriately treated. Performing unnecessary tests and procedures can carry an increased risk of mortality, thus it is important to be aware of post-surgical changes and their complications both on physical examination and imaging studies. ${ }^{8}$

\section{References}

1. Kerr WF. Late-onset post-pneumonectomy empyema. Thorax 1977;32:149-54.

2. Fuentes, P. Pneumonectomy: historical perspective and prospective insight. European Journal of Cardio-thoracic Surgery 2003; 23: 439-445

3. Fuentes PA. Pneumonectomy: historical perspective and prospective insight. Eur J Cardiothorac Surg 2003;23:439-45.
4. Baue AE. Landmark perspective: Evarts A. Graham and the first pneumonectomy. JAMA 1984;251:260-4.Horn, L., Johnson, D., Evarts, A. Graham and the first pneumonectomy for lung cancer. J Clin Oncol. 2008; 26(19):3268-75.

5. Horn L, Johnson DH. Evarts A. Graham and the first pneumonectomy for lung cancer. J Clin Oncol 2008;26:3268-75.

6. Kopec SE, Irwin RS, Umali-Torres CB, Balikian JP, Conlan AA. The postpneumonectomy state. Chest 1998;114:1158-84.

7. Smulders SA, van den Bosch HC, Post JC, Vonk Noordegraaf A, Postmus PE. Where is the heart after left-sided pneumonectomy? J Thorac Oncol 2006;1:69-70.

8. Jordan S, Mitchell JA, Quinlan GJ, Goldstraw P, Evans TW. The pathogenesis of lung injury following pulmonary resection. Eur Respir J 2000;15:790-9.

9. Kopec SE, Conlan AA, Irwin RS. Perforation of the right ventricle: a complication of blind placement of a chest tube into the postpneumonectomy space. Chest 1998;114:1213-5.

10. Jansen JP, Brutel de la Riviere A, Alting MP, Westermann CJ, Bergstein PG, Duurkens VA. Postpneumonectomy syndrome in adulthood. Surgical correction using an expandable prosthesis. Chest 1992;101:1167-70.

11. Macare van Maurik AF, Stubenitsky BM, van Swieten HA, Duurkens VA, Laban E, Kon M. Use of tissue expanders in adult postpneumonectomy syndrome. J Thorac Cardiovasc Surg 2007;134:608-12.

12. Reed MF, Lewis JD. Thoracoscopic mediastinal repositioning for postpneumonectomy syndrome. J Thorac Cardiovasc Surg 2007;133:264-5.

13. Regnard JF, Pouliquen E, Magdeleinat P, et al. [Postpneumonectomy syndrome in adults: description and therapeutic propositions apropos of 8 cases]. Rev Mal Respir 1999;16:1113-9.

14. Lauckner ME, Beggs I, Armstrong RF. The radiological characteristics of bronchopleural fistula following pneumonectomy. Anaesthesia 1983;38:452-6.

15. Tsukada G, Stark P. Postpneumonectomy complications. AJR Am J Roentgenol 1997;169:1363-70

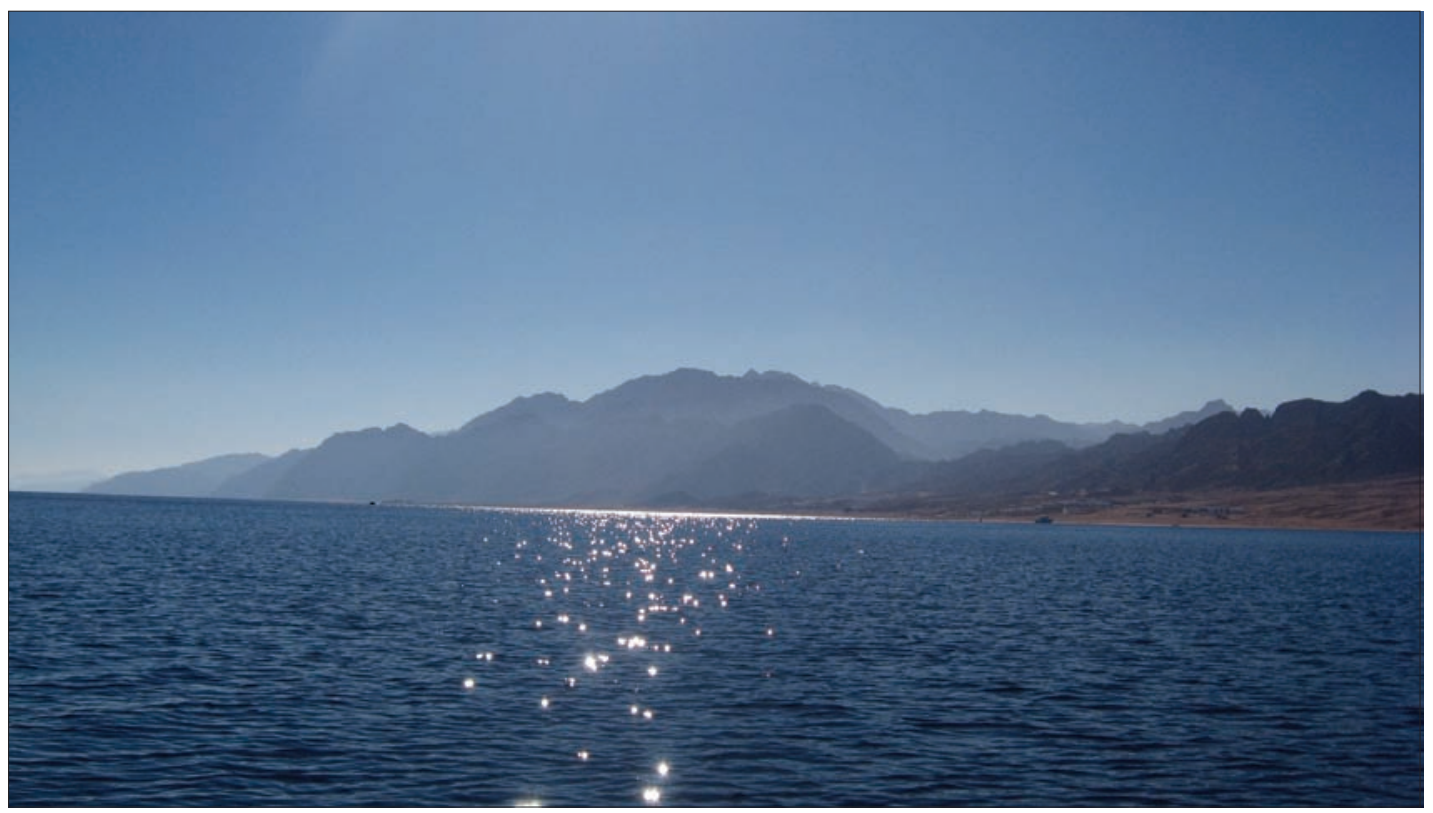

